\title{
Gibbs states on loop lattices: existence and a priori estimates
}

\author{
Sergio ALBEVERIO a, c, Yuri KONDRATIEV b, c, d, \\ Tatiana PASUREK ${ }^{b, c}$, Michael RÖCKNER ${ }^{b, c}$

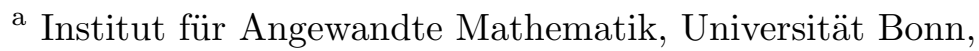 \\ 53155 Bonn, Germany \\ E-mail: albeverio@uni-bonn.de \\ ${ }^{\mathrm{b}}$ Fakultät für Mathematik, Universität Bielefeld, \\ 33615 Bielefeld, Germany \\ E-mail: kondrat@mathematik.uni-bielefeld.de \\ roeckner@mathematik.uni-bielefeld.de \\ ${ }^{c}$ Research Centre BiBoS, Universität Bielefeld, \\ 33615 Bielefeld, Germany \\ E-mail: pasurek@physik.uni-bielefeld.de \\ ${ }^{\mathrm{d}}$ Institute of Mathematics, Kiev, Ukraine
}

\section{Running title: Gibbs states on loop lattices}

\begin{abstract}
We prove existence and uniform a priori estimates for Euclidean Gibbs states of quantum lattice systems with unbounded spins. These results substantially extend all previous existence results. Detailed proofs are contained in [3].

États de Gibbs sur des réseaux de lacets: existence et estimations à priori

Résumé. Des estimations à priori et l'existence des états de Gibbs pour des systèmes quantiques sur réseaux avec spins non bornés sont données. Ces résultats étendent substanciellement tous les résultats d'existence précédents. Les preuves détaillées sont contenues dans [3].
\end{abstract}




\section{Version française abrégée}

Nous étudions un système quantique d'oscillateurs anharmoniques (de masse $\mathfrak{m}>0$ et avec spins non bornés $x_{k} \in \mathbb{R}$ ) distribués sur le réseau $\mathbb{Z}^{d}$. Le système est décrit par un Hamiltonien $\mathbb{H}$ donné heuristiquement par la formule (1) ci-dessous. Les interactions à deux points sont données par des fonctions symétriques qui satisfont à une condition de croissance polynomiale. Les autointeractions harmoniques sont données par $\frac{1}{2} a^{2} x_{k}^{2}$ avec intensité $a>0$. Les autointeractions anharmoniques $V_{k} \in C_{b, l o c}^{2}(\mathbb{R} \rightarrow \mathbb{R})$ satisfont à une condition de coercivité et à une condition de restriction de croissance. Ces conditions sont satisfaites dans le cas de nombreuses interactions d'importance physique.

Nous décrivons les propriétés d'équilibre de ces systèmes quantiques au moyen des états de Gibbs qui sont donnés par des mesures de Gibbs («euclidiennes》) $\mu_{\beta}$ associées avec $\mathbb{H}$ et une température inverse $\beta>0[1,2]$. La définition rigoureuse de $\mu_{\beta}$ est comme suit. Soit $S_{\beta} \cong[0, \beta]$ le cercle avec mesure de Lebesgue $d \tau$. La variable de spin associée à $k \in \mathbb{Z}^{d}$ prend ses valeurs dans $L_{\beta}^{r}:=L^{r}\left(S_{\beta}, d \tau\right), r \geq 1$, resp. $\quad\left(C_{\beta}^{\alpha}=C^{\alpha}\left(S_{\beta}\right), \alpha \geq 0\right) C_{\beta}=C\left(S_{\beta}\right)$ (espaces de lacets $\omega_{k}: S_{\beta} \rightarrow \mathbb{R}$ intégrables resp. (Hölder) continus). Les espaces $\Omega_{\beta}^{-p, r}$ resp. $C_{\beta}^{-\beta, \alpha}$ sont définis ci-dessous. Les sous-ensembles des configurations resp. distributions tempérées sont donnés par $\Omega_{\beta}^{t}:=\cup_{p \geq 1} \Omega_{\beta}^{-p, R}$ resp. $\mathcal{M}_{\beta}^{t}:=\left\{\mu \mid \exists p=p(\mu)>d / 2: \mu\left(\Omega_{\beta}^{-p, R}\right)=1\right\}$ (pour un $R$ approprié). Les mesures de Gibbs $\mu_{\beta}$ sont décrites par leur specification locale $\left\{\pi_{\beta, \Lambda} \mid \Lambda \subset \subset Z^{d}\right\}$ (cf. [4,5]). $\pi_{\beta, \Lambda}$ est défini comme noyau stochastique sur $\left(\Omega_{\beta}^{t}, B\left(\Omega_{\beta}^{t}\right)\right): \forall B \in$ $\mathcal{B}\left(\Omega_{\beta}^{t}\right):=\mathcal{B}\left(\left(C_{\beta}\right)^{Z^{d}}\right) \cap \Omega_{\beta}^{t}, \forall \xi \in \Omega_{\beta}^{t}$ et est donné par (3), (4) ci-dessous. Nous définissons l'ensemble $\mathcal{G}_{\beta}^{t}$ d'états de Gibbs tempérées comme les $\mu_{\beta} \in \mathcal{M}_{\beta}^{t}$ qui satisfont aux équations DLR $\mu_{\beta} \pi_{\beta, \Lambda}=\mu_{\beta}$.

Proposition 1 donne l'identité de $\mathcal{G}_{\beta}^{t}$ avec $\mathcal{M}_{b}^{t}$, un ensemble de measures qui consiste d'éléments $\mu$ dans $\mathcal{M}_{\beta}^{t}$ qui satisfont à la formule d'integration par parties

$$
\int_{\Omega_{\beta}} \partial_{i} f d \mu=-\int_{\Omega_{\beta}} f b_{i} d \mu
$$

pour tous les $f$ appartenant à un certain sous-ensemble de fonctions dans $\left(C_{\beta}\right)^{\mathbb{Z}^{d}}$, et pour toutes les directions $h_{i}$ dans une base orthonormale de $\mathcal{H}_{\beta}:=l^{2}\left(\mathbb{Z}^{d} \rightarrow\right.$ $\left.L_{\beta}^{2}\right)$. $b_{i}$ est la dérivée logarithmique partielle dans la direction $h_{i}$.

Le théorème 1 montre que $\mathcal{G}_{\beta}^{t} \neq \emptyset$. Le théorème 2 montre que chaque $\mu_{\beta} \in \mathcal{G}_{\beta}^{t}$ a son support dans un ensemble de lacets Höldériens, et des estimations de ces moments sont données. Les preuves utilisent un analogue infini-dimensionnel de la méthode des fonctions de Lyapunov. Les résultats contiennent comme cas particuliers tous les résultats connus sur l'existence et les estimations à priori des états de Gibbs euclidiens pour les crystaux quantiques. De plus, le cas d'une croissance superquadratique des potentiels d'integration est inclu. 


\section{Quantum crystals and Euclidean Gibbs mea- sures}

We study an interacting system of quantum anharmonic oscillators (of mass $\mathfrak{m}>$ 0 and with unbounded spins $\left.x_{k} \in \mathbb{R}\right)$ on a lattice $\mathbb{Z}^{d}\left(\subset \mathbb{R}^{d}\right), d \in \mathbb{N}$, which is described by the heuristic Hamiltonian

$$
\mathbb{H}:=-\frac{1}{2 \mathfrak{m}} \sum_{k \in \mathbb{Z}^{d}} \frac{d^{2}}{d x_{k}^{2}}+\frac{a^{2}}{2} \sum_{k \in \mathbb{Z}^{d}} x_{k}^{2}+\sum_{\{k, j\} \subset \mathbb{Z}^{d}} W_{\{k, j\}}\left(x_{k}, x_{j}\right)+\sum_{k \in \mathbb{Z}^{d}} V_{k}\left(x_{k}\right),
$$

(see e.g. [6] for physical motivations). We specify the assumptions on the system (1) as follows:

$\mathbf{A}_{1}$ ) The two-particle interactions (taken over all unordered pairs $\{k, j\} \subset \mathbb{Z}^{d}$, $k \neq j)$ are given by symmetric functions $W_{\{k, j\}} \in C_{b, p o l}^{2}\left(\mathbb{R}^{2} \rightarrow \mathbb{R}\right)$ satisfying the polynomial growth condition: $\exists R \geq 2, \exists J_{\{k, j\}} \geq 0, \forall x_{k}, x_{j} \in \mathbb{R}$

$$
\left|\partial_{k}^{(l)} W_{\{k, j\}}\left(x_{k}, x_{j}\right)\right| \leq J_{\{k, j\}}\left(1+\left|x_{k}\right|+\left|x_{j}\right|\right)^{R-l}, \quad l=0,1,2,
$$

where $\partial_{k}^{(l)}$ denotes the $l$-th derivative w.r.t. coordinate $x_{k}$.

$\mathbf{A}_{2}$ ) The matrix $J=\left\{J_{\{k, j\}}\right\}$ is fastly decreasing, that is, $\forall p \in \mathbb{N}$ :

$$
\|J\|_{p}:=\sup _{k \in \mathbb{Z}^{d}}\left\{\sum_{j \in \mathbb{Z}^{d} \backslash\{k\}} J_{\{k, j\}}^{2}(1+|k-j|)^{2 p}\right\}^{1 / 2}<\infty .
$$

$\left.\mathbf{A}_{3}\right)$ The harmonic self-interactions are given by $a^{2} x_{k}^{2} / 2$ with an intensity $a>0$. The anharmonic self-interactions $V_{k} \in C_{b, l o c}^{2}(\mathbb{R} \rightarrow \mathbb{R})$ satisfy the coercivity estimate: $\exists A_{1}, B_{1}, \sigma>0, \forall k \in \mathbb{Z}^{d}, \forall x_{k} \in \mathbb{R}$

$$
V_{k}^{\prime}\left(x_{k}\right) x_{k} \geq A_{1}^{-1}\left[\left|x_{k}\right|^{R+\sigma}+\left|V_{k}^{\prime}\left(x_{k}\right)\right|+\left|V_{k}^{\prime \prime}\left(x_{k}\right) x_{k}\right|\right]-B_{1}
$$

and the growth condition: $\exists A_{2}, B_{2}>0, \forall k \in \mathbb{Z}^{d}, \forall x_{k} \in \mathbb{R}$

$$
\left|V_{k}^{\prime \prime}\left(x_{k}\right)\right| \leq A_{2}\left[\left|x_{k}\right|^{R-1}+\left|V_{k}^{\prime}\left(x_{k}\right)\right|\right]+B_{2} .
$$

We note that the above assumptions are fulfilled for many classes of interactions of physical relevance.

A mathematical description of equilibrium properties of quantum systems is carried out in terms of their temperature (i.e., Gibbs) states. We will take the Euclidean (i.e., path space) approach, see e.g. $[1,2]$ and the references therein. Therewith the Euclidean Gibbs measures $\mu_{\beta}$ associated with the lattice system (1) at the inverse temperature $\beta>0$ are rigorously defined as follows: 
Let $S_{\beta} \cong[0, \beta]$ be a circle with Lebesgue measure $d \tau$. As the single spin spaces for every $k \in \mathbb{Z}^{d}$ we will use the spaces $L_{\beta}^{r}:=L^{r}\left(S_{\beta}, d \tau\right), r \geq 1$, resp. $\left(C_{\beta}^{\alpha}:=C^{\alpha}\left(S_{\beta}\right), \alpha \geq 0\right) C_{\beta}:=C\left(S_{\beta}\right)$ of integrable resp. (Hölder) continuous loops $\omega_{k}: S_{\beta} \longrightarrow \mathbb{R}$. As the configuration space for the infinite volume system (1) we define the temperature loop lattices

$$
\begin{aligned}
& \Omega_{\beta}^{-p, r}:=\left\{\left.\omega \in\left(C_{\beta}\right)^{\mathbb{Z}^{d}}\left|\|\omega\|_{-p, r}^{2}:=\sum_{k \in \mathbb{Z}^{d}}(1+|k|)^{-2 p}\right| \omega_{k}\right|_{L_{\beta}^{r}} ^{2}<\infty\right\}, \\
& \mathcal{C}_{\beta}^{-p, \alpha}:=\left\{\left.\omega \in\left(C_{\beta}^{\alpha}\right)^{\mathbb{Z}^{d}}\left|\|\omega\|_{-p, \alpha}^{2}:=\sum_{k \in \mathbb{Z}^{d}}(1+|k|)^{-2 p}\right| \omega_{k}\right|_{C_{\beta}^{\alpha}} ^{2}<\infty\right\}
\end{aligned}
$$

Thereafter, we define the subsets of tempered configurations resp. of tempered distributions by

$$
\Omega_{\beta}^{t}:=\bigcup_{p \geq 1} \Omega_{\beta}^{-p, R} \text { resp. } \mathcal{M}_{\beta}^{t}:=\left\{\mu \mid \exists p=p(\mu)>d / 2: \mu\left(\Omega_{\beta}^{-p, R}\right)=1\right\} .
$$

The Euclidean Gibbs measures $\mu_{\beta}$ are described by their local specifications $\left\{\pi_{\beta, \Lambda} \mid \Lambda \Subset \mathbb{Z}^{d}\right\}$ (cf. $[4,5]$ ). Let $\gamma_{\beta}$ be a centered Gaussian measure on $C_{\beta}$ with correlation operator $\mathbb{A}_{\beta}^{-1}$, where $\mathbb{A}_{\beta}:=-\mathfrak{m} d^{2} / d \tau^{2}+a^{2} \mathbf{1}$ is considered as the self-adjoint operator in the Hilbert space $L_{\beta}^{2}$. For $\Lambda \Subset \mathbb{Z}^{d}, \pi_{\beta, \Lambda}$ is defined as a stochastic kernel on $\left(\Omega_{\beta}^{t}, \mathcal{B}\left(\Omega_{\beta}^{t}\right)\right): \forall B \in \mathcal{B}\left(\Omega_{\beta}^{t}\right):=\mathcal{B}\left(\left(C_{\beta}\right)^{\mathbb{Z}^{d}}\right) \cap \Omega_{\beta}^{t}, \forall \xi \in \Omega_{\beta}^{t}$

$$
\pi_{\beta, \Lambda}(B \mid \xi):=Z_{\beta, \Lambda}^{-1}(\xi) \int_{\Omega_{\beta, \Lambda}} \exp \left\{-\mathcal{I}_{\beta, \Lambda}^{V, W}(\omega \mid \xi)\right\} \mathbf{1}_{\Delta}\left(\omega_{\Lambda}, \xi_{\Lambda^{c}}\right) \prod_{k \in \Lambda} d \gamma_{\beta}\left(\omega_{k}\right)
$$

Here $\omega_{\Lambda}:=\left(\omega_{k}\right)_{k \in \Lambda} \in\left(C_{\beta}\right)^{\Lambda}, Z_{\beta, \Lambda}(\xi)$ is a normalization factor, and

$$
\mathcal{I}_{\beta, \Lambda}^{V, W}(\omega \mid \xi):=\int_{S_{\beta}}\left[\sum_{k \in \Lambda} V_{k}\left(\omega_{k}\right)+\sum_{\{k, j\} \subset \Lambda} W_{\{k, j\}}\left(\omega_{k}, \omega_{j}\right)+\sum_{k \in \Lambda, j \in \Lambda^{c}} W_{\{k, j\}}\left(\omega_{k}, \xi_{j}\right)\right] d \tau
$$

Obviously, (3), (4) make sense because of $\left(\mathbf{A}_{1-3}\right)$. We define the set $\mathcal{G}_{\beta}^{t}$ of tempered Euclidean Gibbs states as those $\mu \in \mathcal{M}_{\beta}^{t}$ which satisfy the DLR equations $\mu_{\beta} \pi_{\beta, \Lambda}=\mu_{\beta}, \forall \Lambda \Subset \mathbb{Z}^{d}$.

\section{Main results}

We start with an integration by parts (IbP) description of $\mu_{\beta} \in \mathcal{G}_{\beta}^{t}$. We fix the orthonormal basis $h_{i}:=\left\{\delta_{k-j} \varphi_{n}\right\}_{j \in \mathbb{Z}^{d}}, i=(k, n) \in \mathbb{Z}^{d+1}$ in $\mathcal{H}_{\beta}:=l^{2}\left(\mathbb{Z}^{d} \rightarrow\right.$ 
$L_{\beta}^{2}$ ), where $\left\{\varphi_{n}\right\}_{n \in \mathbb{Z}} \subset C_{\beta}^{\infty}$ is respectively the complete orthonormal system of eigenvectors of the operator $\mathbb{A}_{\beta}$ in $L_{\beta}^{2}$ (i.e., $\mathbb{A}_{\beta} \varphi_{n}=\lambda_{n} \varphi_{n}$ with $\lambda_{n}=(2 \pi n / \beta)^{2} \mathfrak{m}+$ $\left.a^{2}\right)$. $\mathbb{Z}^{d+1}$

We define the partial logarithmic derivatives along directions $h_{i}, i=(k, n) \in$

$$
b_{i}(\omega):=-\left(\mathbb{A}_{\beta} \varphi_{n}, \omega_{k}\right)_{\beta}-\left(F_{k}^{V, W}(\omega), \varphi_{n}\right)_{\beta}, \quad \omega \in \Omega_{\beta}^{t},
$$

where $F_{k}^{V, W}: \Omega_{\beta}^{t} \rightarrow L_{\beta}^{1}$ is given by

$$
F_{k}^{V, W}(\omega):=V_{k}^{\prime}\left(\omega_{k}\right)+\sum_{j \neq k} \partial_{k} W_{\{k, j\}}\left(\omega_{k}, \omega_{j}\right)
$$

Fixed $i=(k, n) \in \mathbb{Z}^{d+1}, p>d / 2$, we define the set $C_{\mathrm{dec}, i}^{1}\left(\Omega_{\beta}^{-p, R_{W}}\right)$ of all functions $f: \Omega_{\beta}^{-p, R_{W}} \rightarrow \mathbb{R}$ which are bounded and continuous together with their partial derivatives $\partial_{i} f:=\partial_{h_{i}} f$ in the direction $h_{i}$ and, moreover, fit the extra decay condition

$$
|f(\omega)| \leq C_{k}(f)\left(1+\left|\omega_{k}\right|_{L_{\beta}^{1}}+\left|F_{k}^{V, W}(\omega)\right|_{L_{\beta}^{1}}\right)^{-1}, \omega \in \Omega_{\beta}^{-p, R_{W}} .
$$

Proposition 1 (IbP Characterization) Let $\mathcal{M}_{b}^{t}$ denote the set of all probability measures $\mu$ on $\Omega_{\beta}^{t}$ which for some $p>d / 2$ satisfy the temperedness condition (2) and the (IbP)-formula

$$
\int_{\Omega_{\beta}} \partial_{i} f(\omega) d \mu(\omega)=-\int_{\Omega_{\beta}} f(\omega) b_{i}(\omega) d \mu(\omega)
$$

for all functions $f \in C_{\mathrm{dec}, i}^{1}\left(\Omega_{\beta}^{-p, R_{W}}\right)$ and directions $h_{i}, i=\mathbb{Z}^{d+1}$. Then $\mathcal{G}_{\beta}^{t}=\mathcal{M}_{b}^{t}$.

The measures given by the local specifications $\pi_{\beta, \Lambda}$ satisfy Propositions 1 , but only in directions $h_{i}, i=(k, n), k \in \Lambda \Subset \mathbb{Z}^{d}, n \in \mathbb{Z}$. Since under our assumptions $b_{i}$ are continuous locally bounded functions on $\Omega_{\beta}^{-p, R_{W}}$, the latter means that every accumulation point of the family $\left\{\pi_{\beta, \Lambda}(d \omega \mid \xi) \mid \Lambda \Subset \mathbb{Z}^{d}, \xi \in \Omega_{\beta}^{t}\right\}$ is Gibbs.

Theorem 1 (Existence of Tempered Gibbs States) Let assumptions ( $\mathbf{A}_{1-3}$ ) on the potentials $V_{k}$ and $W_{\{k, j\}}$ be fulfilled. Then for all values of mass $\mathfrak{m}>0$ and temperature $\beta>0$

$$
\mathcal{G}_{\beta}^{t} \neq \emptyset
$$


Theorem 2 (A Priori Estimates on Tempered Gibbs States) Let assumptions $\left(\mathbf{A}_{1-3}\right)$ be fulfilled. Then every $\mu_{\beta} \in \mathcal{G}_{\beta}^{t}$ is supported by the set of Hölder loops $\bigcap_{\substack{p>d / 2 \\ 0 \leq \alpha<1 / 2}} \mathcal{C}_{\beta}^{-p, \alpha}$. Moreover, $\forall Q \geq 1$

$$
\text { (i) } \sup _{\substack{\mu_{\beta} \in \mathcal{G}_{\beta}^{t} \\ k \in \mathbb{Z}^{d}}} \int_{\Omega_{\beta}}\left|\omega_{k}\right|_{C_{\beta}^{\alpha}}^{Q} d \mu_{\beta}(\omega)<\infty,
$$

and thus

$$
\text { (ii) } \sup _{\substack{\mu_{\beta} \in \mathcal{G}_{\beta}^{t} \\ \tau \in S_{\beta}, k \in \mathbb{Z}^{d}}} \int_{\Omega_{\beta}}\left|\omega_{k}(\tau)\right|^{Q} d \mu_{\beta}(\omega)<\infty \text {. }
$$

The key point of the proofs is that (according to Proposition 1) $\mu_{\beta}$ resp. $\pi_{\beta, \Lambda}$ are viewed as the solutions of an infinite system of first order partial differential equations. Due to the pointwise coercivity and growth assumptions $\left(\mathbf{A}_{1-3}\right)$ on the potentials $V_{k}, W_{\{k, j\}}$, the corresponding vector fields $b_{i}, i \in \mathbb{Z}^{d+1}$, also possess certain coercivity properties w.r.t. the tangent space $\mathcal{H}_{\beta}$. This allows us to apply an analog of the Lyapunov function method well known from finite dimensional PDE's (cf. [3]).

\section{Concluding remarks}

Among the particular novelities of our results are the following:

1) We emphasize that Theorems 1, 2 include all known results on the existence and a priori estimates for Euclidean Gibbs states for quantum crystals as particular cases.

2) The case of superquadratic growth of the interaction potentials is included. Note that this case does not admit an application of the well-known superstability bounds of Ruelle [7].

3) Many-particle interactions can be considered in the same way (cf. [3]). Also the case $\sigma=0$ in assumption $\left(\mathbf{A}_{3}\right)$ is allowed by taking constant $A_{1}>0$ small enough.

4) The a priori estimates described above are uniform w.r.t. all Gibbs measures from $\mathcal{G}_{\beta}^{t}$.

The precise relation to already known results is discussed in [3] in detail.

Acknowledgements. Financial support by the DFG through SFB 237 BochumDüsseldorf-Essen, SFB 343 Bielefeld as well as the Research Project AL 214/17-2, and by the EC-Science Project SC1*CT92-0784 is gratefully acknowledged. 


\section{References}

[1] Albeverio S., Kondratiev Yu. G., Röckner M., TsikalenkoT. V., Uniqueness of Gibbs states for quantum lattice systems, Prob. Theory Related Fields 108 (1997), 193-218.

[2] Albeverio S., Kondratiev Yu. G., Röckner M., Tsikalenko T. V., Glauber dynamics for quantum lattice systems, Rev. Math. Phys. 13 (2001), 51-124.

[3] Albeverio S., Kondratiev Yu. G., Röckner M., Pasurek (Tsikalenko) T., In preparartion (2001).

[4] Dobrushin R. L., Prescribing a system of random variables by conditional distributions, Theory Prob. Appl. 15 (1970), 458-486.

[5] Georgii H.-O., Gibbs measures and phase transitions, Studies in Mathematics, 9, Walter de Gruyter, Berlin-New York, 1988.

[6] Minlos R. A, Verbeure A., Zagrebnov V. A., A quantum crystal model in the light mass limit: Gibbs state, Rev. Math. Phys. 12 (2000), 981-1032.

[7] Ruelle D., Probability estimates for continuous spin systems, Comm. Math. Phys. 50 (1976), 189-194. 